\title{
Milliampere Beam Studies using High Polarization Photocathodes at the CEBAF Photoinjector
}

\author{
Joseph Grames ${ }^{1}$, D. Moser, P. Adderley, J. Hansknecht, R. Kazimi, M. Poelker, \\ M. Stutzman, R. Suleiman, S. Zhang \\ Thomas Jefferson National Accelerator Facility \\ 12000 Jefferson Ave., Newport News, VA 23606 USA \\ E-mail: gramesejlab.org
}

\begin{abstract}
Extending the charge lifetime of today's spin polarized GaAs photoelectron guns from tens to thousands of Coulombs is a requirement for long-duration operation at milliampere beam current. There are two main approaches frequently considered to achieve this goal. The first is improving photo-gun vacuum, which provides a direct means to minimize the ill effects of ion bombardment and associated QE decay. In this contribution, we demonstrate the second: enhancing the charge lifetime at milliampere beam current by using large laser spots which serves to distribute ion damage over a larger region of the photocathode. The efficacy of this approach was presented at past workshops, but using bulk GaAs and green laser light without RF structure. In this contribution, highly polarized beam was produced from strained-superlattice GaAs/GaAsP photocathodes inside the $130 \mathrm{kV}$ photo-gun at the CEBAF photoinjector, at beam currents from 0.5 to $1.5 \mathrm{~mA}$ and with a clear indication of lifetime enhancement using laser beams as large as $\sim 8 \mathrm{~mm}^{2}$. However, when the laser beam size was increased beyond this diameter, further lifetime enhancement was not observed which highlights the importance of proper cathode/anode design to maintain loss-free beam delivery and the preservation of static vacuum conditions. In addition, this contribution presents electron beam polarization measurements at currents to $1 \mathrm{~mA}$.
\end{abstract}

XVII International Workshop on Polarized Sources, Targets \& Polarimetry

16-20 October 2017

Kaist, South Korea 


\section{Introduction}

GaAs photocathodes inside dc high voltage photo-guns are the primary means to produce spin polarized electron beams for nuclear and high energy physics experiments at particle accelerators. To be useful, such photocathodes must exhibit sufficiently long photocathode operating lifetime in order to sustain the required beam current for an experimental program. For example, the GaAs/GaAsP strained-superlattice photocathode in the CEBAF $130 \mathrm{kV}$ photo-gun has routinely exhibited an operating charge lifetime of $\sim 250 \mathrm{C}$, and therefore is capable of providing beam currents up to $\sim 200 \mu \mathrm{A}(\sim 20 \mathrm{C} /$ day $)$ for $3-4$ weeks without intervention. In contrast, in order to sustain a beam current $>1 \mathrm{~mA}(\sim 86 \mathrm{C} /$ day $)$ for a similar duration, the photocathode charge lifetime should exceed $1000 \mathrm{C}$, which has never been demonstrated.

This contribution describes three studies recently performed at the Jefferson Lab CEBAF injector to better understand the operation of high polarization $\mathrm{GaAs} / \mathrm{GaAsP}$ strained-superlattice photocathodes at beam currents exceeding $1 \mathrm{~mA}$. First, the use of x-ray and vacuum gauges to monitor the effect of low level beam loss on photocathode lifetime was studied. Second, the ability to increase the charge lifetime by increasing the laser spot size was applied. Finally, the first measurement of electron spin polarization of a milliampere beam at Jefferson Lab was demonstrated, using an $\mathrm{MeV}$ Mott polarimeter.

\subsection{The Experiment}

The beam line used for these measurements is shown in Fig. 1. The electron beam exits the photo-gun through a large bore ( 2.5 inch diameter) NEG-coated beam tube towards a $15^{\circ}$ bend magnet. This bend allows illumination of the photocathode at normal incidence without using mirrors inside the vacuum chamber. Five solenoid magnets ( $\sim 50 \mathrm{~cm}$ ) and air core steering magnets were used to transport the beam $\sim 7 \mathrm{~m}$ to a Faraday cup. A differential NEG pump station provides beam line vacuum isolation from the Faraday Cup. The gun and beam line vacuum are monitored by precision ion pump power supplies with a resolution of $\sim 50 \mathrm{pA}$. Geiger Muller (GM) tubes positioned along the beam line beginning at the exit of the photo-gun adjacent to limiting apertures, such as the entrance to a Wien filter and an RF buncher cavity were used to detect beam loss.

Make beam here

$(-130 \mathrm{kV}, 0-1.5 \mathrm{~mA}, 0-3 \mathrm{pC})$

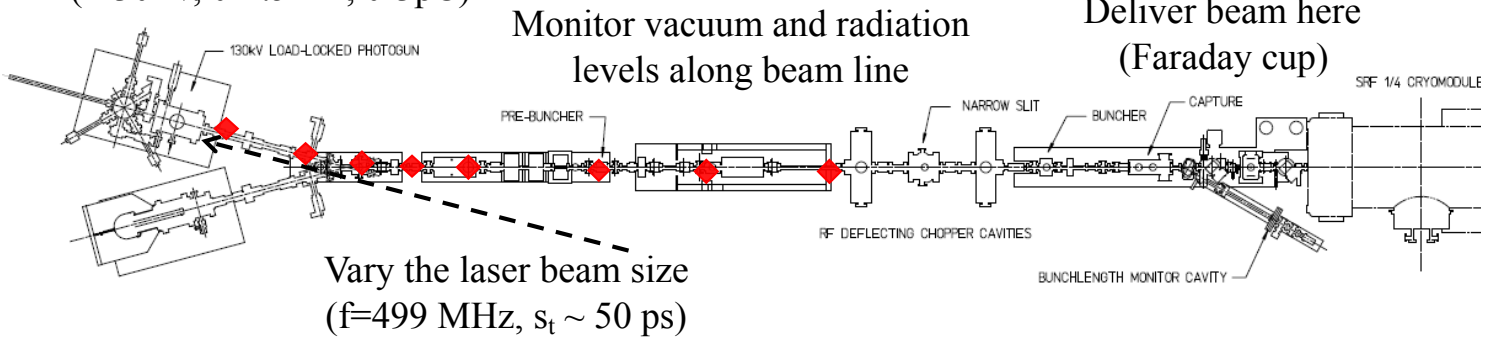

Fig. 1. The figure shows the region of the CEBAF injector where high current studies were performed. The (red) diamonds indicate where GM tubes were positioned.

A fiber-based laser produced light at $780 \mathrm{~nm}$, the value for peak polarization, with $\sim 50 \mathrm{ps}$ optical pulses and $499 \mathrm{MHz}$ pulse repetition rate (i.e., CEBAF conditions). The laser spot size at 
the photocathode was defined by inserting a pair of lenses with appropriate focal length upstream of the beam line vacuum window. The laser intensity profile was measured by diverting the laser beam to an optical profile analyzer located at the equivalent photocathode distance. The spot size was varied over a broad range from $3-20 \mathrm{~mm}^{2}$ (see Fig. 2). The laser intensity was remotely controlled using a polarization sensitive attenuator with high extinction ratio. The laser power was measured continuously via a pick-off laser beam directed into a power meter, calibrated to an inline power meter allowing for continuous monitoring of the photocathode quantum efficiency.

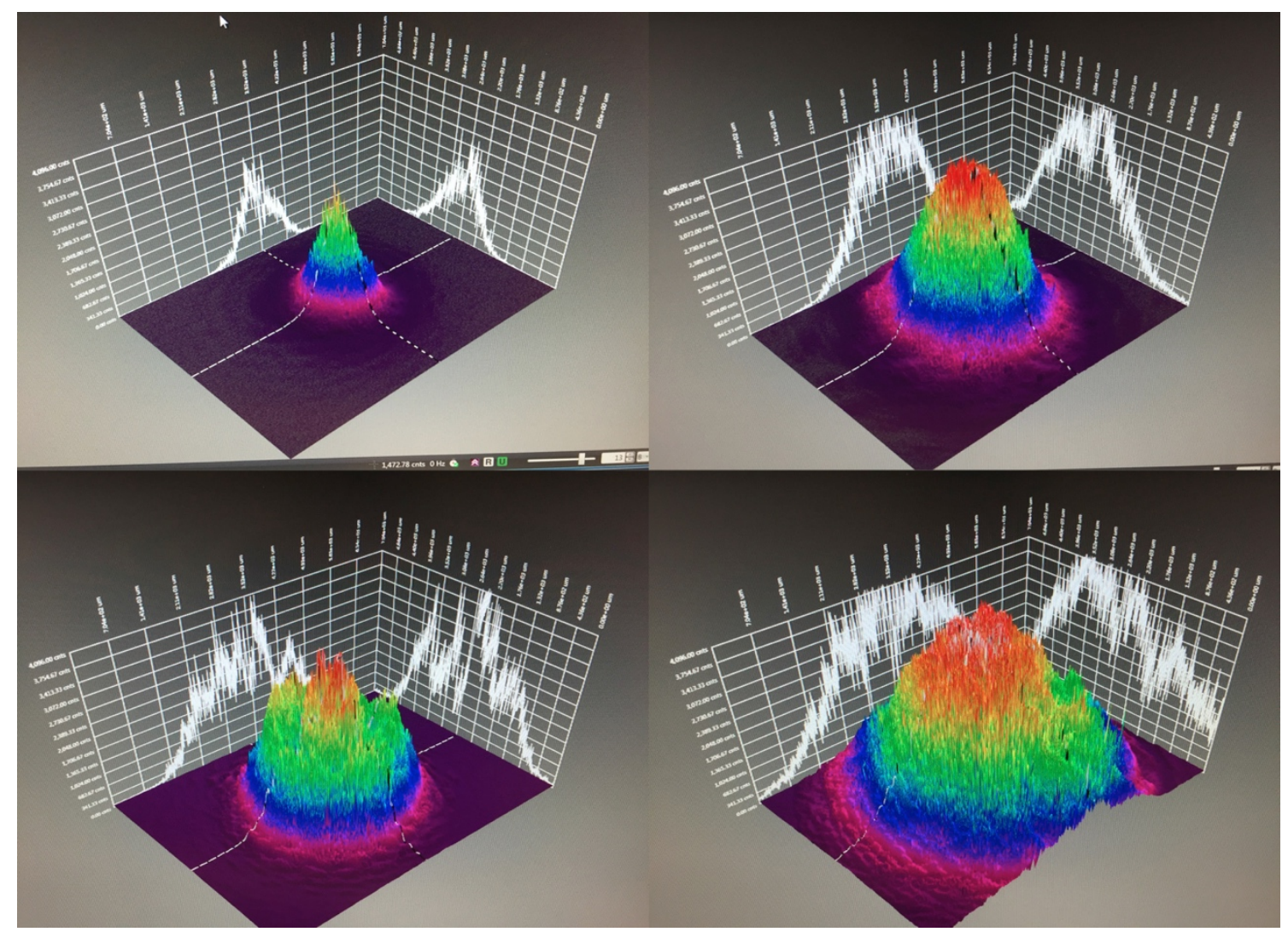

Fig. 2. Measurements of the laser profiler were made for sizes ranging from $\sigma_{4 \mathrm{D}} \sim 1-5 \mathrm{~mm}$.

\subsection{Detecting Low Level Beam Loss}

The vacuum level when delivering beam is often greater than the static level achieved when the beam is off. This dynamic vacuum degradation can reasonably be attributed to gas desorption associated with electrons striking the beamline surfaces or the bremsstrahlung x-rays resulting from it. The use of precision ion pumps to detect beam loss induced gas desorption is a proven tool to assess photocathode charge lifetime [1], however, future gains in photocathode lifetime may come with the ability to detect beam loss that does not exceed the effective static "noise floor" of even very good beam line vacuum. In order to improve our sensitivity to beam loss, Geiger Muller (GM) tubes were installed along the CEBAF injector photo-gun beam line. The sensitivity of the GM tube to beam loss was studied by purposely "dumping" known amounts of beam current $(0.5,5,50 \mathrm{nA})$ along the beam line using steering coils. 

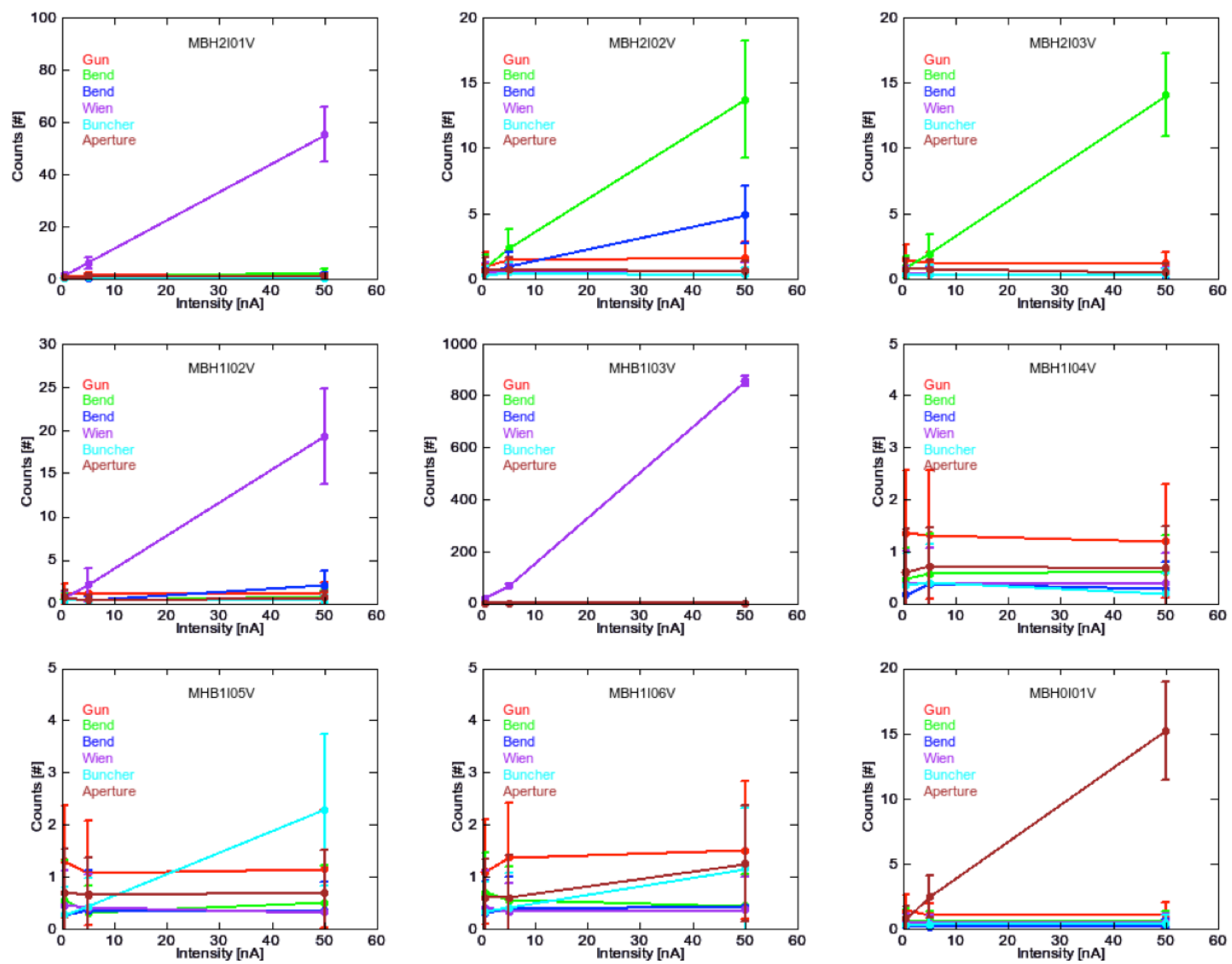

Fig. 3. The GM tube count rates vs. beam current was measured when the beam was "dumped" along the beam line. The slope of the line indicates the sensitivity to beam loss. Generally one GM tube was most sensitive (largest slope).

The results of the study are presented in Fig. 3, showing the measured GM tube radiation levels as a function of beam current for nine locations where a steering coil "dumped" the beam. Although the absolute count rate is dependent on the details of each GM tube position, and vacuum components that shield radiation (e.g., the thickness of the beam pipe, flanges), the GM tubes provide a useful relative signal when calibrated to absolute beam loss. Generally, one GM tube is most sensitive to each steering coil suggesting the tubes are useful for localized monitoring. The resolution of sensitivity (1 count) typically corresponded to 1-5 nA of lost beam current. However, the greatest sensitivity corresponded to $\sim 60 \mathrm{pA}$, suggesting a more judicious placement of tubes could generally provide sub-nanoampere beam loss detection.

\subsection{Charge Lifetime at High Average Current}

Charge lifetime enhancement of bulk GaAs photocathodes by increasing the laser spot size has been previously demonstrated [2]. The same technique was used successfully to enhance the charge lifetime of GaAs/GaAsP strained-superlattice photocathode at CEBAF, where the beam charge lifetime when providing $\sim 200 \mu \mathrm{A}$ for the Qweak experiment [3] was increased a factor of four by doubling the laser spot diameter. In this contribution we extend this work to higher beam currents and with larger laser spot sizes. The 1/e charge lifetimes measured for milliampere beam currents $(1-1.5 \mathrm{~mA})$ as a function of the illuminated area of the photocathode are summarized in Fig. 4. By increasing the laser spot size from $\sim 3 \mathrm{~mm}^{2}$ to $\sim 8 \mathrm{~mm}^{2}$ (2.6 times) 
the average charge lifetime increased by the expected factor, from $\sim 150 \mathrm{C}$ to $\sim 350 \mathrm{C}$ ( $\sim 2.8$ times). In particular, for the case of $1 \mathrm{~mA}$ the charge lifetime was measured in excess of $400 \mathrm{C}$, the highest value reported to date at milliampere beam current. For this condition, one could expect to operate for about one week before the initial QE drops to $\sim 1 / 10^{\text {th }}\left(1 / \mathrm{e}^{2}\right)$ of the original value. Interestingly, however, the charge lifetime did not improve as the laser spot size was increased further. One speculation is the increased transverse emittance of the beam due to the increased laser spot size exceeded the useful acceptance of the gun and beam line, becoming increasingly sensitive to the geometric aberrations of the cathode/anode electrostatic field and/or the magnetic lenses used to transport the beam. This claim is supported by previous measurements [2] that demonstrated the dependence of charge lifetime on the radial position of the laser beam at the photocathode. Note that in the present studies the laser spot size was tested well beyond the expected typical operating value $\left(<2.5 \mathrm{~mm}^{2}\right)$, up to the full extent of the active area $\left(19 \mathrm{~mm}^{2}\right)$.

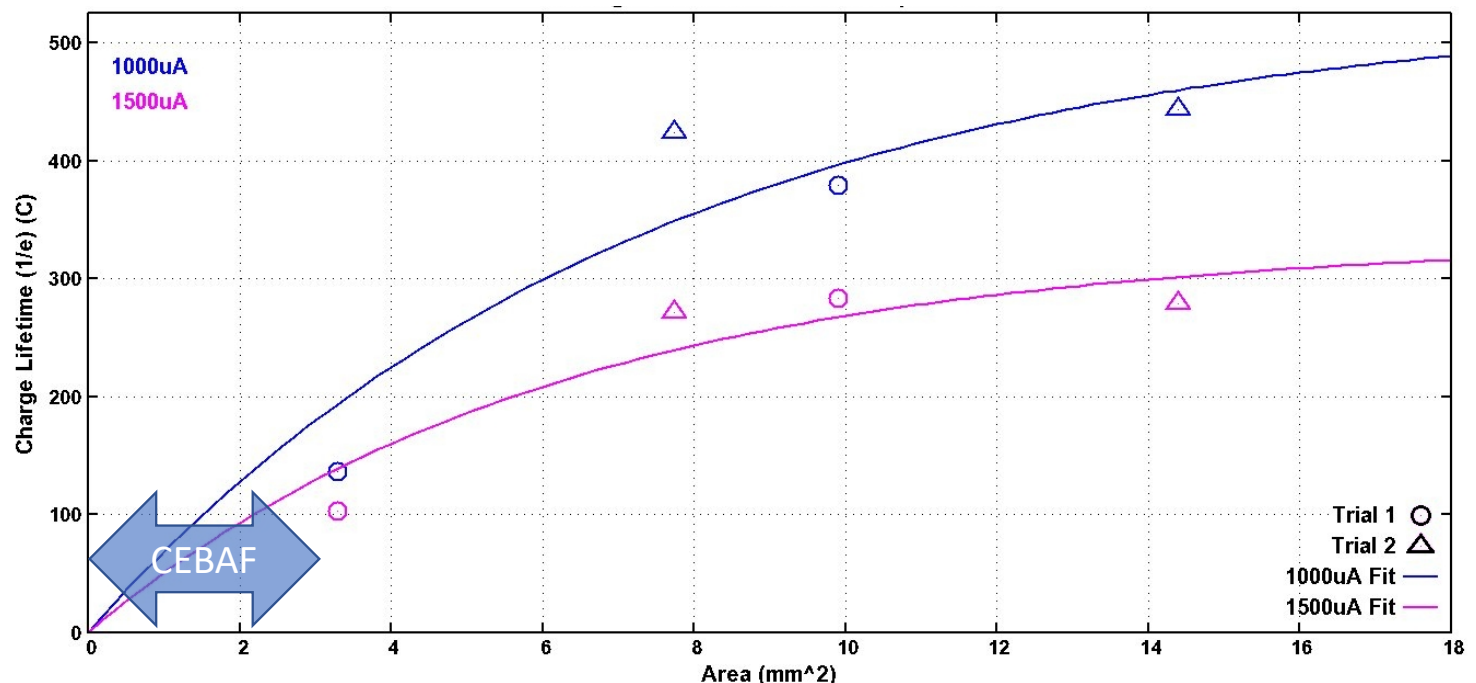

Fig. 4. Charge lifetime of GaAs/GaAsP at CEBAF $130 \mathrm{kV}$ photogun for milliampere current as a function of laser spot area.

To investigate why charge lifetime did not scale as expected, for laser areas $>8 \mathrm{~mm}^{2}$, the GM tubes were used to monitor radiation levels during charge lifetime runs that span broader laser spot size and beam currents $(0.5-1.5 \mathrm{~mA})$, with a focus on detecting beam loss. The correlation plots of radiation level versus charge lifetime (see Fig. 5) indicate that even very low levels of radiation associated with beam loss serve to adversely impact charge lifetime. It must be noted that this level of beam loss was not detected by sensitive ion pump power supplies previously used to validate accelerator setup. In this study, the best charge lifetimes - approaching $\sim 500 \mathrm{C}$ - were associated with GM tubes detecting beam loss below the $\sim$ nanoamp level, corresponding to beam loss of less than $\sim 1 \mathrm{ppm}$. 

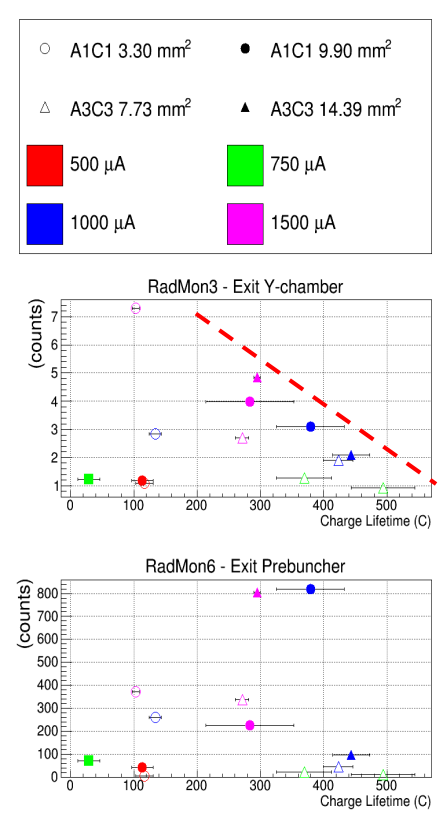
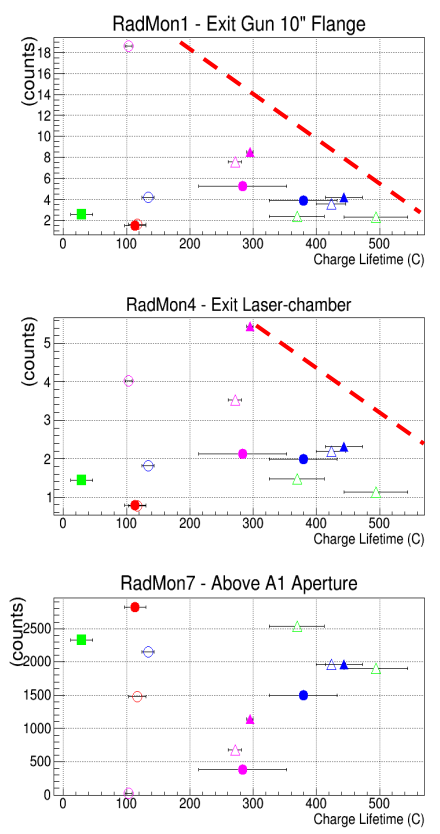
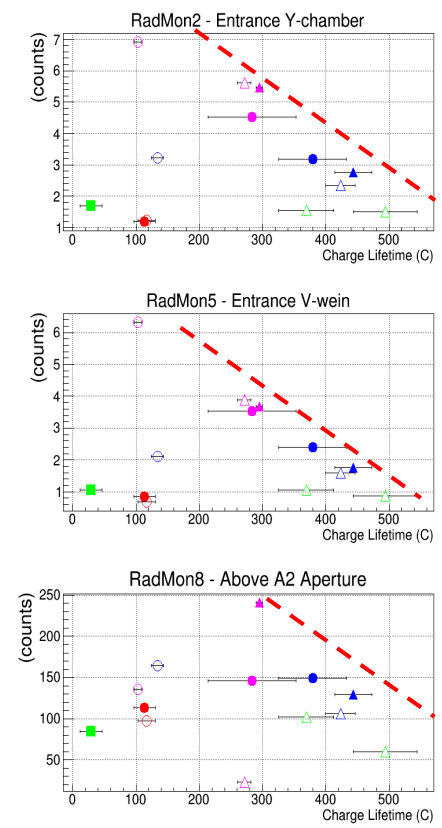

Fig. 5. Correlation plots of the run-average GM tube count signal as a function of the corresponding charge lifetime. The dashed line (same relative slope in each) is included to make the point of increased charge lifetime with decreased radiation level.

\subsection{Spin Polarization at High Average Current}

In the final study, the beam polarization of a GaAs/GaAsP strained-superlattice photocathode operating at beam current up to one milliampere was measured. To facilitate the measurement, the electron beam was accelerated in the CEBAF injector (see Fig. 6) to $\sim 6 \mathrm{MeV}$ and deflected into a Mott polarimeter with a precisely known analyzing power. Owing to the large Mott scattering cross-section and the resultant large scattering rate $(\sim 500 \mathrm{~Hz} / \mathrm{uA})$, a beam current of just $1 \mu \mathrm{A}$ is sufficient for rapid statistically significant measurements $(<0.5 \%)$. To provide this current at the polarimeter, a variable temporal aperture was used to pass only a portion of the beam current produced at the photo-gun. The result of the study is shown in Fig. 7, with beam current varied from 1 to $1000 \mu \mathrm{A}$, and polarization essentially constant (polarization $\sim 85 \%$ ) within error bars.

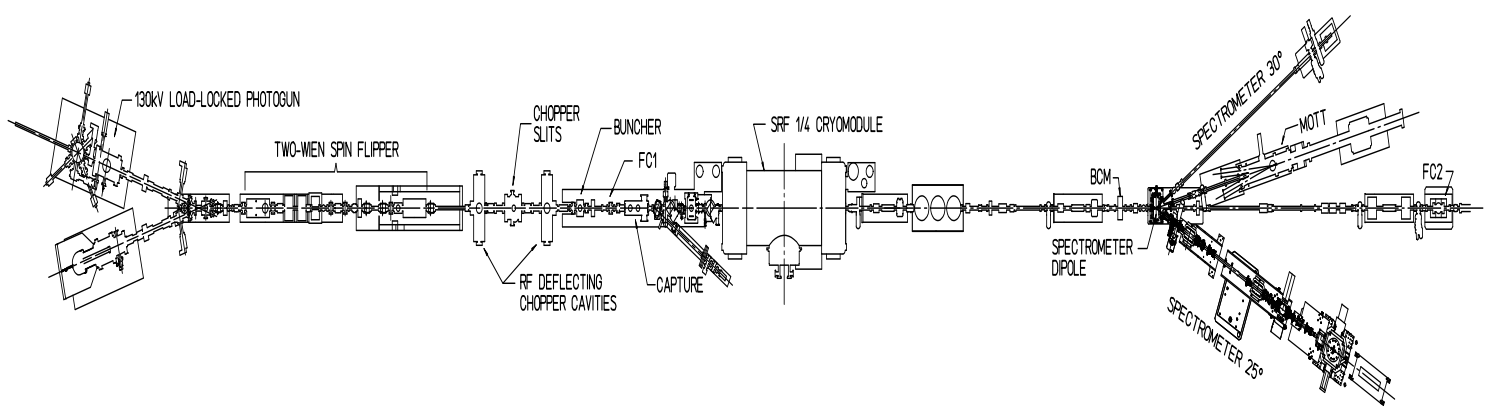

Fig. 6. Spin polarization measurements are made at the CEBAF injector after accelerating the beam to $\sim 6 \mathrm{MeV}$ and using a Mott scattering polarimeter. 


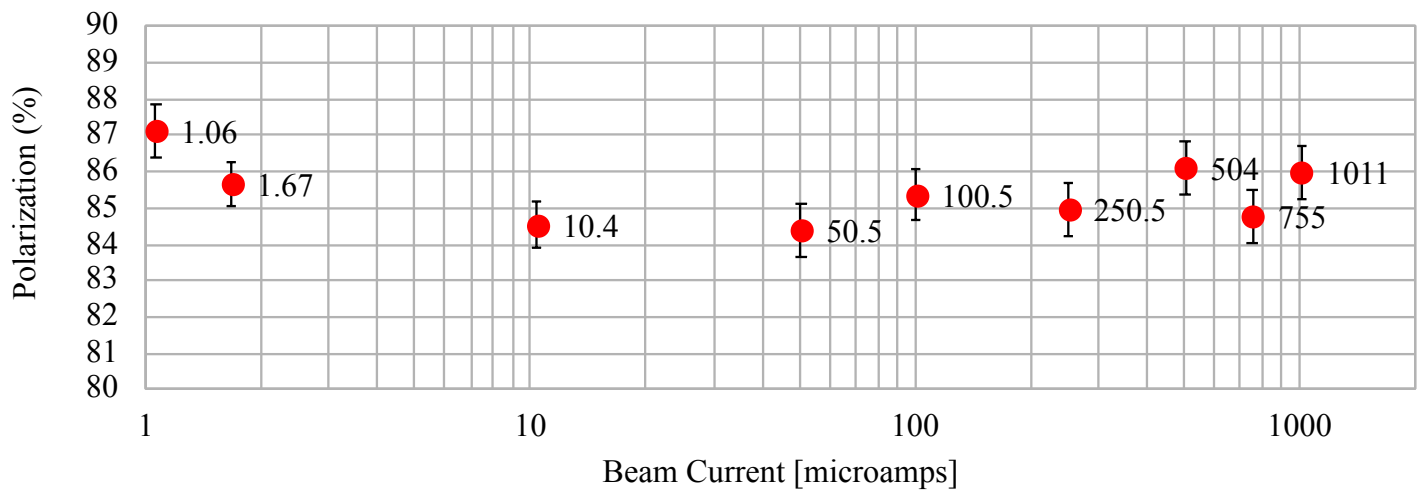

Fig. 7. Spin polarization measurements of a GaAs/GaAsP photocathode demonstrate high spin polarization spanning three decades of beam current from $1 \mu \mathrm{A}$ to $1 \mathrm{~mA}$.

\subsubsection{Conclusions}

These measurements represent the first milliampere beam studies with $\mathrm{GaAs} / \mathrm{GaAsP}$ strained-superlattice photocathode at the CEBAF injector. By increasing the laser spot size, a charge lifetime of $>400 \mathrm{C}$ was obtained, representing the highest charge lifetime value ever reported for a highly polarized electron beam at milliampere beam current. In addition, the electron spin polarization was measured and found to be nearly constant $(\sim 85 \%)$ from $1 \mathrm{uA}$ to 1 $\mathrm{mA}$.

Geiger Muller tubes were used to detect beam loss with demonstrated detectable resolution of $\sim 60 \mathrm{pA}$, representing a significant improvement over beam loss monitoring using vacuum gauges (i.e., the ion pump current). Measurements indicate beam loss at the part-per-million level is signficant, adversely effecting charge lifetime, and limiting ones ability to enhance charge lifetime by simply increasing the laser spot size indefintiely. For the CEBAF photogun, laser beams greater than $8 \mathrm{~mm}^{2}$ resulted in unacceptable beam loss. Further studies will be pursued to extend the capability of the photo-gun to test scaling for larger laser spot size as a pathway to generate $\sim \mathrm{kC}$ charge lifetime at milliampere beam currents.

\section{References}

[1] C. K. Sinclair, M. Poelker, P. A. Adderley, B. M. Dunham, J. C. Hansknecht, P. Hartmann, J. S. Price, P. M. Rutt, W. J. Schneider, and M. Steigerwald, "A High Average Current Polarized Electron Source with Long Cathode Operational Lifetime", Phys. Rev. ST Accel. Beams 10, 023501 (20077)

[2] J. Grames, R. Suleiman, P. A. Adderley, J. Clark, J. Hansknecht, D. Machie, M. Poelker, and M. L. Stutzman, "Charge and fluence lifetime measurements of a DC high voltage GaAs photogun at high average current", Phys. Rev. ST Accel. Beams 14, 043501 (2011)

[3] Jefferson Lab Qweak Collaboration. "Precision measurement of the weak charge of the proton" Nature 557, 207 